\title{
Rehabilitación estética del sector anterior con cerámica libre de metal. Reporte de
}

\section{caso.}

\author{
Esthetic rehabilitation of the anterior sector with metal-free ceramic. Case Report. \\ César Lamas-Lara ${ }^{1, a}$, Sergio Alvarado-Menacho ${ }^{2, b}$, Giselle Angulo de la Vega ${ }^{3, c}$.
}

\section{RESUMEN}

Los requerimientos estéticos en la odontología actual son bastante altos por parte de nuestros pacientes y los profesionales deben estar capacitados para ofrecer las mejores alternativas técnicas y procedimentales. Además el profesional debe de estar preparado en las diferentes áreas de la odontología para así identificar los procedimientos más adecuados a realizar. El objetivo de éste trabajo enfocó el manejo multidisciplinario en la rehabilitación del sector anterosuperior con la participación de las especialidades de endodoncia, periodoncia y rehabilitación oral. Los resultados obtenidos fueron satisfactorios para el paciente ya que se logró rehabilitar adecuadamente las piezas dentales involucradas desde un punto de vista estético y funcional. Concluimos que en la odontología actual en donde la estética prima en nuestros tratamientos el profesional debe de estar capacitado para poder saber elegir los materiales más adecuados para sus tratamientos sin descuidar los aspectos funcionales.

\section{Palabras clave: REHABILITACIÓN BUCAL, GINGIVOPLASTÍA, CORONAS DENTALES.}

\begin{abstract}
The current dental aesthetic requirements are quite high by our patients and professionals must be able to offer the best technical and procedural alternatives. Also the professional must be prepared in different areas of dentistry to thus identify the most appropriate procedures to carry out. The aim of this work focused on the multidisciplinary handling in the rehabilitation of the anterosuperior sector with the participation of the specialties of Endodontics, Periodontics and oral rehabilitation. The results obtained were satisfactory for the patient since it managed to properly rehabilitate the teeth involved from an aesthetic and functional point of view. We conclude that current dentistry where aesthetic prevails in our treatments professional must be able to to know how to choose the most appropriate materials for their treatments without neglecting functional aspects.
\end{abstract}

\section{Key Words: MOUTH REHABILITATIONS, GINGIVOPLASTY, DENTAL CROWNS.}

\footnotetext{
${ }^{1}$ Facultad de Odontología de la Universidad de San Martín de Porres. Lima, Perú.

2 Facultad de Odontología de la Universidad Nacional Mayor de San Marcos. Lima, Perú.

${ }^{3}$ Facultad de Odontología de la Universidad Científica del Sur. Lima, Perú.

a Cirujano Dentista, Diplomado en Odontología Restauradora y Estética

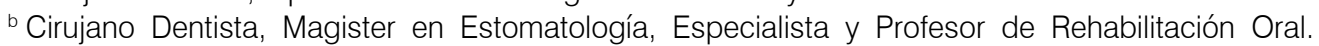

c Cirujano Dentista, Estudios de Especialidad en Rehabilitación Oral.
} 


\section{INTRODUCCIÓN}

El primer paso para realizar un adecuado tratamiento restaurador es un adecuado diagnóstico; para realizar esto es fundamental un enfoque multidisciplinario para obtener todas las perspectivas necesarias. La visión de una sola especialidad en odontología puede llevar a no apreciar las soluciones clínicas más adecuadas para nuestros pacientes; y además este tratamiento sólo estará completo cuando se eduque al paciente para realizar un mantenimiento y así lograr obtener su salud periodontal $(1,2)$.

Los usos e indicaciones de las coronas metal cerámicas han sido ampliamente descritos y son considerados como una buena opción en la rehabilitación de los pacientes ya que cuentan con una alta predictibilidad $(3,4)$.

En la actualidad los materiales cerámicos han evolucionado rápidamente y esto obliga al profesional a capacitarse de manera adecuada para ofrecer a nuestros pacientes las diferentes opciones con las que contamos para lograr los mejores resultados posibles (5).

Las coronas de cerámica libres de metal pueden fabricarse de diferentes cerámicas vítreas y cerámicas convencionales. Entre estas cerámicas se distinguen los siguientes grupos principales:

a) Cerámica vítrea de óxido de silicio (cerámica vítrea de dióxido de silicio, cerámica vítrea de silicato o cerámica vítrea de feldespato).

b) Cerámica de óxido de aluminio.

c) Cerámica de óxido de zirconio (también denominada cerámica de dióxido de zirconio).

Estos materiales pueden modificarse y procesarse de distintas maneras. Naturalmente existen otras cerámicas que también son aptas para su aplicación técnica, sin embargo no son adecuadas en odontología por su color (6).

La realización correcta del tratamiento de nuestros pacientes parte de un adecuado diagnóstico y para ello debemos de valernos de todas las ayudas diagnósticas como son el uso de radiografías, fotografías, modelos de estudios correctamente articulados, etc. Y el articulador semiajustable nos ayuda a evaluar con claridad la oclusión de nuestros paciente $(7,8)$.

En el sector anterior los postes de fibra de vidrio nos ofrecen no solamente buenas propiedades estéticas u ópticas sino la literatura nos demuestra sus buenas propiedades mecánicas y funcionales, y esta postura es defendida por varios autores $(9,11)$.

La aplicación de los procedimientos de periodoncia en rehabilitación nos ayuda a mejorar las condiciones para que nuestros tratamientos obtengan resultados más predecibles; así podemos mejorar las condiciones iniciales del tratamiento. Es por ello que se hace necesario el conocer y emplear estos diferentes procedimientos $(12,13)$.

El objetivo del caso presentado fue realizar una adecuada rehabilitación de las piezas dentarias del sector anterosuperior devolviendo así la estética y la función perdida a través del manejo odontológico multidisciplinario.

\section{REPORTE DEL CASO}

Paciente de sexo femenino de 20 años de edad que acude a la consulta odontológica preocupada por su problema estético en el sector anterosuperior. La paciente refiere haber sufrido un traumatismo dentoalveolar hace 10 años comprometiendo el sector anterosuperior razón por la cual le realizaron una reconstrucción y ferulización con resina compuesta.

Al examinar a la paciente se puedó apreciar la presencia de las piezas dentarias 1.1 y 2.1 con restauraciones extensas con resinas compuesta, cambio de color y ferulizadas. También se puedo apreciar el engrosamiento de la encía vestibular a ese nivel. (fig. 1)

A nivel de las piezas 1.3, 1.2, 2.2 y 2.3 presentaban restauraciones defectuosas con presencia de microfiltración marginal.

Se realizó la toma de radiografías periapicales y la toma de modelos de estudio, su posterior articulado para el correcto análisis de la oclusión.

Se realizaron las pruebas de vitalidad pulpar obteniendo una respuesta negativa a nivel de las piezas 1.1 y 2.1. 


\section{Diagnóstico}

Del estado de salud general: Paciente con buen estado de salud general, sin riesgo sistémico al tratamiento estomatológico.

Del estado de salud estomatológico: Paciente con:

1. Necrosis Pulpar a nivel de las piezas 1.1 y 2.1

2. Caries dental: caries recidivante a nivel de las piezas dentarias $1.3,1.2,2.2$ y 2.3

\section{Plan de Tratamiento}

Paciente si riesgo al tratamiento estomatológico:

1. Fisioterapia oral, enseñanza de la técnica de cepillado, hilo dental y enjuague bucal.

2. Necropulpectomía: en las piezas dentarias 1.1 y 2.1

3. Colocación de postes preformados de fibra de vidrio en las piezas 1.1 y 2.1

4. Gingivoplastía a nivel de las piezas 1.3, 1.2, 1.1, $2.1,2.2$ y 2.3

5. Confección de Coronas cerámicas en las piezas $1.3,1.2,1.1,2.1,2.2$ y 2.3

6. Reforzamiento de la enseñanza de la técnica de cepillado, uso del hilo dental y enjuague bucal para el mantenimiento adecuado de la rehabilitación protésica.

\section{TRATAMIENTO}

Se procedió a reforzar la técnica de cepillado, del uso del hilo dental y del enjuague bucal.

Luego de la aceptación del paciente de los procedimientos que se iban a realizar, se procedió a realizar los tratamientos de endodoncia y la colocación de los postes de fibra de vidrio en las piezas 1.1 y 2.1. Se le explicó a la paciente que una alternativa sería poder realizar procedimientos más conservadores como son las reconstrucciones con resinas compuestas o carillas de cerámica para devolver la estética en el sector anterior, pero la paciente optó por realizarse coronas libres de metal en las 6 piezas dentales por considerar un mejor efecto de grupo y mayor predictibilidad en el resultado final.

Posteriormente se procedió a realizar el tallado de las piezas del sector anterosuperior y la confección de provisionales directos (fig 2).
Analizando el sector anterosuperior (figura 3) podemos apreciar que el largo de los dientes no guarda armonía de grupo, por consiguiente se realizó una gingivoplastía para mejora los largos de las piezas dentarias y un retallado inmediato (fig 4).

Se realizaron nuevos provisionales y se evaluó los resultados obtenidos logrando resultados satisfactorios (fig 5).

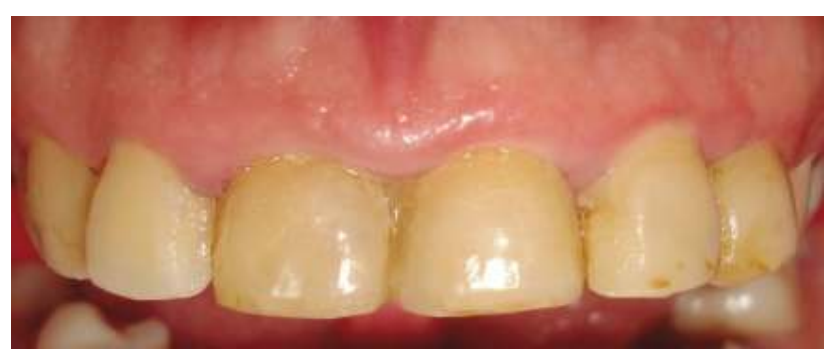

Fig 1. Fotografia Inicial.

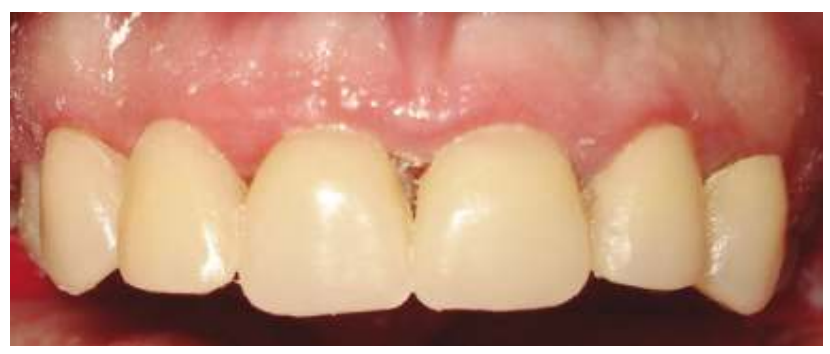

Fig 2. Primer tallado y confección de Provisionales.

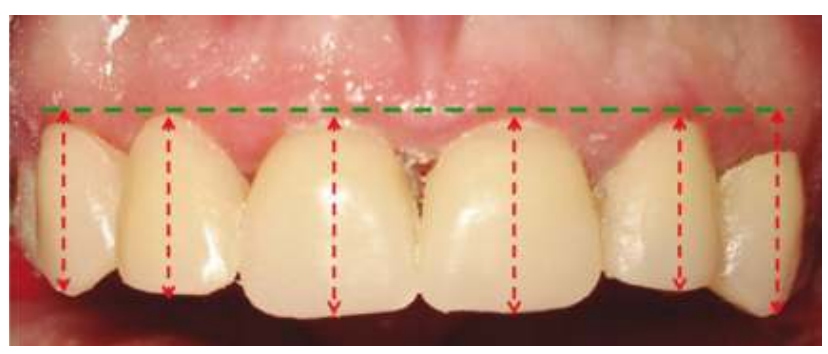

Fig 3. Planificación para la cirugía periodontal.

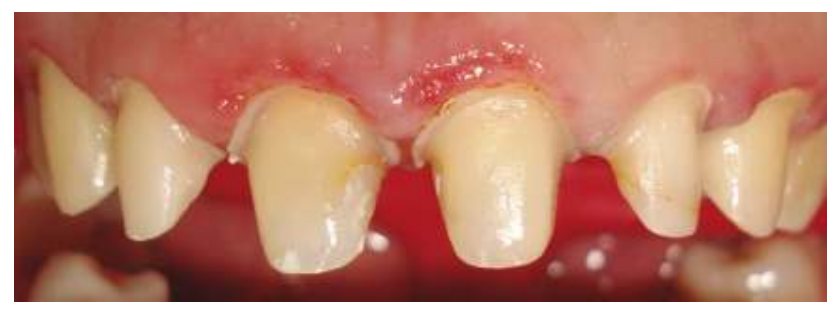

Fig 4. Confección de nuevos Provisionales. 


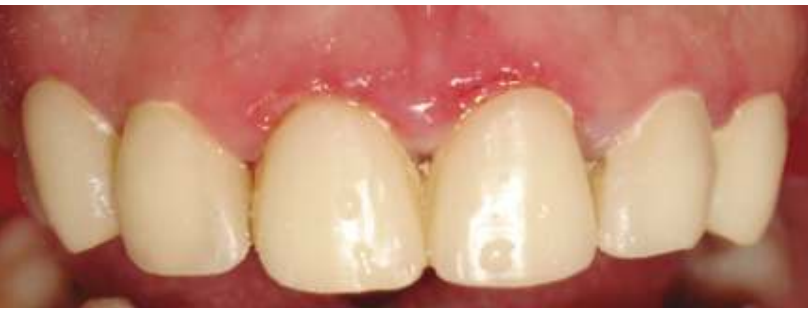

Fig 5. Confección de nuevos Provisionales.

Luego de esperar 2 semanas, se realizó la toma de impresión definitiva con silicona por adición para obtener los modelos de trabajo y se envió al laboratorio para la confección de las coronas de cerámica sin metal (fig 6). Se seleccionó el color A1 en la escala VITA.

Una vez realizadas las pruebas en boca del paciente y con su aceptación, se procedió a realizar el cementado de las coronas libres de metal con un cemento de ionómero de vidrio (fig 7).

Inmediatamente después del cementado de la coronas se puedó apreciar la ausencia de las papilas interdentales y se le explica al paciente que a medida que pase el tiempo este espacio va a ser ocupado por la encía formando una nueva papila.

Se le pide al paciente que acuda a sus controles periódicos (fig 8), en la cual se puedó apreciar la neoformación de las papilas interproximales (fig 9 y 10).

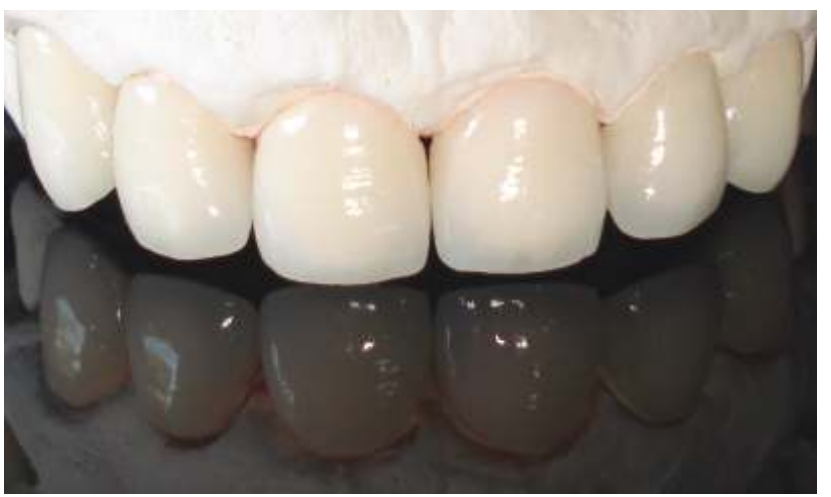

Fig 6. Coronas Definitivas

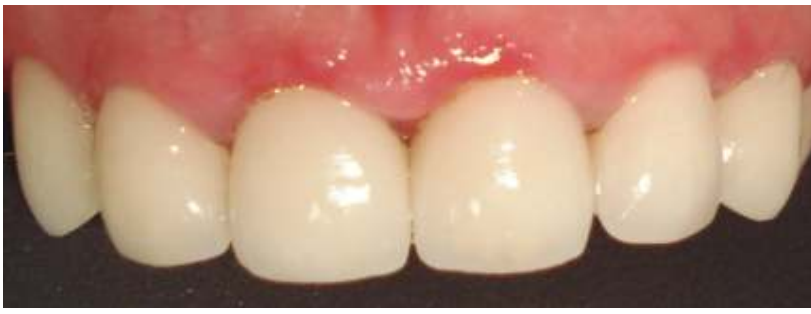

Fig 7. Cementación definitiva.

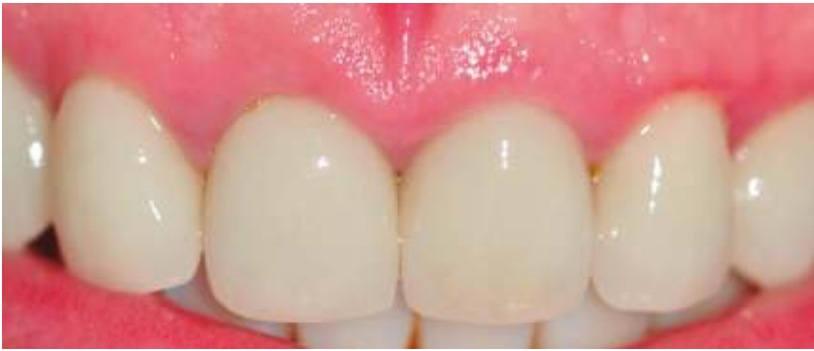

Fig 8. Fotografía de control 4 años después.

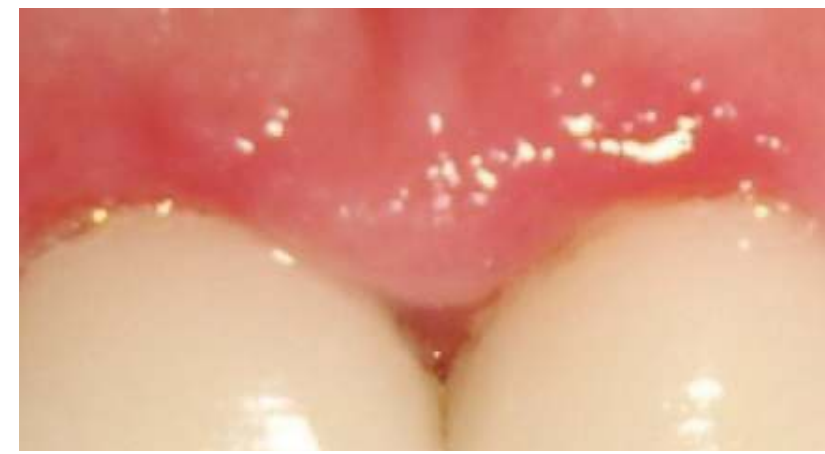

Fig 9. Vista de la Encía inmediatamente después de terminado el tratamiento.

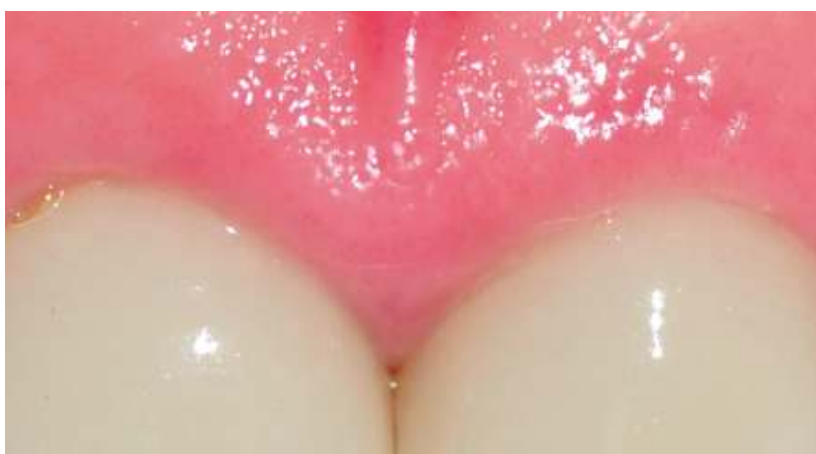

Fig 10. Vista de la encía 4 años después de terminado el tratamiento. 


\section{RESULTADOS}

Los resultados obtenidos fueron satisfactorios para la paciente ya que se logró rehabilitar adecuadamente las piezas dentarias involucradas devolviendo la estética y función perdida.

Al devolver adecuadamente los contactos interproximales en el sector anterior se produjo una neoformación de la papila, de esta manera los triangulo oscuros que se formaban por la ausencia de estas papilas se redujo al mínimo.

\section{DISCUSIÓN}

Las prótesis fijas metal cerámicas presentan una excelente biocompatibilidad y buenas propiedades mecánicas, muchos estudios demuestran la duración de los tratamientos en donde este material fue utilizado; es por ello que en la actualidad su uso se hace muy frecuente. Pero uno de sus principales inconvenientes es su limitada estética en especial a nivel cervical y en una población donde cada vez más los pacientes exigen tratamientos estéticos, esta situación se vuelve imperiosa y surge la necesidad de buscar materiales que nos brinden mejores posibilidades en este aspecto. Es por ello que el uso de las coronas de cerámica libres de metal surge como una posibilidad válida y muy aconsejable para este tipo de situaciones clínicas ya que presentan buenas propiedades tanto ópticas como mecánicas $(13,18)$.

\section{CONCLUSIONES}

El mejorar las condiciones de salud periodontal de las piezas dentarias que vamos a rehabilitar nos garantiza la longevidad de nuestras restauraciones, así mismo es fundamental que el paciente pueda mantener una adecuada salud periodontal.

Una adecuada planificación basada en un buen diagnóstico es fundamental para realizar un buen tratamiento, es por ello que el profesional debe de estar preparado para poder identificar toda la patología que se pueda presentar y realizar las interconsultas correspondientes a las demás especialidades asociadas para realizar un optimo tratamiento en beneficio del paciente.

En la época actual, donde la estética prima en nues- tros tratamientos, el profesional debe estar capacitado para poder saber elegir los materiales y técnicas más adecuadas para realizar sus tratamientos. Las coronas libres de metal han demostrando muy buenos resultados tanto desde el punto de vista estético como funcional.

\section{CORRESPONDENCIA}

\section{César Lamas Lara}

Santa Honorata 415. Urb. Pando, 3era Etapa.

Lima 1- Perú.

Teléfono: 999491403

Correo electrónico: cesar2579@hotmail.com

\section{REFERENCIAS BIBLIOGRÁFICAS}

1. Lamas C, Paz J, Paredes G, Angulo G, Cardozo S. Rehabilitación Integral en Odontología, Odontol. Sanmarquina, 2012;15(1):31-4.

2. Lamas C, Cárdenas M, Angulo G. Tratamiento multidisciplinario en Odontología, In Cres, 2012;3(2):299-306.

3. Brecker CS. Porcelain baked to gold: A new médium in prosthodontics. J. Prosthet. Dent, 1986;23(6):801-811.

4. Romeo M, Martínez JA, Vallejo J. Introducción a los sistemas CAD/CAM de uso en clínica y laboratorio. Rev. Int. Prot. Estomatol. 2005;7(5):524-534.

5. Janda R, Cerámicas sin metal: composición, propiedades, aplicación, valoración Quintessenz Zahntech. 2007;33(1):46-60.

6. Koushyar K. Recomendaciones para la Selección del Material Cerámico Libre de Metal, de Acuerdo a la Ubicación de la Restauración en la Arcada, Int. J. Odontostomat., 2010;4(3):237-240

7. Pegoraro L. F. Prótesis Fija. Sao Paulo, Artes Medicas, 2001.45-7 pp.

8. Gutierrez M, et al. Importancia de la Oclusión Dentaria en la Rehabilitación por Prótesis Parcial Fija. Rev Cubana Estomatol 2001;38(3):155-64.

9. Bertoldi A. Nuevos enfoques en la reconstrucción coronaria del diente endodonciado. Rev Asoc Odont Argent 2002;4(90):157-62.

10.Monticelli F, Grandini S, Goracci C, Ferrari M. Comportamiento clínico de los pernos de fibra traslúcida: estudio prospectivo de 2 años. Revista internacional de prótesis estomatológica 2004;6(4):321-324.

11.Lamas C, Alvarado S, Pari R. Poste Anatómico Preformado, Odontol. Sanmarquina, 2009;12(1):33-5.

12.Agudelo C, Rico J, Molano E. Alargamiento coronal preprotésico: un procedimiento estético y funcional. Reporte de un caso. Rev. Estomat. 2010;18(2):23-2.

13. Villaseñor C, López M. Tratamiento interdisciplinario de paciente con sonrisa gingival: Reporte de un caso. 
Rev. Odontológica Mexicana 2013;17(1):51-6.

14.Vilarrubí A, Pebé P, Rodríguez A. Prótesis fija convencional libre de metal: tecnología CAD CAM-Zirconia, descripción de un caso clínico. Odontoestomatología 2011;13(18):16-28.

15.Suárez MJ, Lozano JF, Salido MP, Martínez F. Threeyear clinical evaluation of In-Ceram Zirconio posterior FPDs. Int J Prosthodont 2004;17(6):35-8.

16.Zarone S, Russo S, Sorrentino R. From porcelainfused-to-metal to zirconia clinical and experimental considerations. Dental Materials 2011;27(1):83-96.
17.Tinschert J, Natt G, Mautsch W. Marginal fi t of aluminaand zirconia-based fi xed partial dentures produced by a CAD/CAM system. Operat Dent 2011;26(4):367-374.

18.Fraxino B, Goulart CR, Caregnatto ME, Pedroso LM, Leite MS. Técnica Indirecta Para Reparación De Prótesis Parcial Fija Metal-Porcelana Relato Del Caso. Acta odontol. venez. 2010;48(1):164-9.

19.Ozcan M. Fracture reasons in ceramic-fused-tometal restorations. Journal of Oral Rehabilitation. 2003;30(3):265-9.

Recibido : 18-08-2013

Aceptado: 25-11-2013 